\title{
Modelos Interventivo-Terapêuticos em Avaliação Psicológica: Estado da Arte no Brasil
}

\author{
Alessandro Antonio Scaduto ${ }^{1}$ \\ Universidade Federal do Paraná, Curitiba-PR, Brasil \\ Lucila Moraes Cardoso \\ Universidade Estadual do Ceará (UECE), Fortaleza-CE, Brasil \\ Vanessa Stumpf Heck \\ Faculdade de Filosofia, Ciências e Letras de Ribeirão Preto - Universidade de São Paulo-SP, Brasil
}

\section{RESUMO}

Diferentes autores vêm descrevendo usos inovadores de recursos de Avaliação Psicológica para promoção de efeitos terapêuticos em Psicologias Clínica e/ou da Saúde. O presente artigo apresenta três modelos nessa direção, a saber, o Psicodiagnóstico Interventivo (PI), o Psicodiagnóstico Interventivo de Orientação Psicanalítica (PIOP) e a Avaliação Terapêutica (Therapeutic Assessment; TA). São apresentados os fundamentos, estrutura (tipo de técnicas e intervenções usadas), vantagens da proposta em relação ao modelo tradicional de Avaliação Psicológica e pesquisas sobre cada modelo, seguido de uma discussão sobre seus potenciais e limitações. Conclui-se que os modelos compartilham entre si a proposta de integrar avaliação e intervenção breves, promovendo efeitos terapêuticos ao proporcionar que as pessoas atendidas sejam acolhidas e tenham um conhecimento ampliado de suas queixas e seu potencial para lidar com elas. Ao mesmo tempo, os três modelos apresentam limitações para o atendimento a queixas de alta gravidade ou em contextos de avaliação compulsória.

Palavras-chaves: avaliação psicológica; avaliação terapêutica; psicodiagnóstico; psicodiagnóstico interventivo; intervenção.

\section{ABSTRACT - Therapeutic and Interventional Models in Psychological Assessment: State of the Art in Brazil}

Several authors have described innovative uses of psychological assessment resources, in order to promote therapeutic effects in Clinical and/or Health Psychology contexts. The present study discusses three of these models, namely, Interventional Psychodiagnosis, Psychoanalytical-based Interventional Psychodiagnosis and Therapeutic Assessment. We present the assumptions, structure (techniques and interventions), advantages compared to traditional assessment models and research in Brazil so far of these models; following this, we discuss the potentials and limitations of these models. We conclude that the three models share the assumption that assessment and brief interventions are integrated, in order to promote therapeutic effects, offering the clients opportunities to feel respected and comprehend their own requirements. At the same time, these models are limited for helping people with severe complaints, or in compulsory assessment situations.

Keywords: psychological assessment; therapeutic assessment; psychodiagnosis; interventional psychodiagnosis; intervention.

\section{RESUMEN - Modelos Intervencionistas Terapéuticos en Evaluación Psicológica: Estado del Arte en Brasil}

Diferentes autores vienen describiendo usos innovadores de recursos de Evaluación Psicológica para promoción de efectos terapéuticos en Psicologías Clínica y/o de la Salud. El artículo presenta tres modelos en esa dirección, el Psicodiagnóstico Intervencionista (PI), el Psicodiagnóstico Intervencionista de Orientación Psicoanalítica (PIOP) y la Evaluación Terapéutica (Therapeutic Assessment; TA). Se presentan los fundamentos, la estructura (tipo de técnicas e intervenciones utilizadas), las ventajas de la propuesta con relación al modelo tradicional de Evaluación Psicológica y las investigaciones sobre cada modelo, seguido de una discusión sobre sus potenciales y sus limitaciones. Se concluye que, los modelos comparten entre sí la propuesta de integrar evaluación e intervención breves, promoviendo efectos terapéuticos al proporcionar que las personas atendidas sean acogidas y tengan un conocimiento mayor de sus quejas y su potencial para lidiar con las mismas. Asimismo, los tres modelos presentan limitaciones para la atención a quejas de alta gravedad o en contextos de evaluación obligatoria.

Palabras clave: evaluación psicológica; evaluación terapéutica; psicodiagnóstico; psicodiagnóstico intervencionista; intervención.

O panorama recente da Avaliação Psicológica (AP) no Brasil pode ser descrito como um renascimento da área, após a superação de diversas limitações e questionamentos (principalmente durante as décadas de 1980 e 1990), relativas à escassez de pesquisas e baixa qualidade, tanto de grande parte dos instrumentos 
em uso quanto da formação básica na área (Borsa, 2016; Santos, Anache, \& Santana, 2015). Nesse contexto, diversos autores vêm descrevendo estudos e práticas mais coesos ao nível técnico-metodológico, contribuindo para a consolidação da AP no país. Ainda que diversos desafios perduram na atualidade, seu amadurecimento pode ser demonstrado pela diversidade e aprimoramento de instrumentos e fundamentos (por exemplo, Damásio e Borsa, 2018; Hutz, Bandeira, Trentini, \& Krug, 2016 e Hutz, Bandeira, \& Trentini, 2018).

Nessa direção, o presente artigo tem por objetivo apresentar e discutir três modelos que, em comum, compartilham da observação de que o uso de recursos da área de AP pode ser usado para promover efeitos terapêuticos. Ainda que, recentemente, Villemor-Amaral (2016) tenha revisado brevemente tais modelos, enfatizando as perspectivas futuras de um deles em particular, propõe-se no presente estudo um aprofundamento dessa comparação. É importante ressaltar que a proposta de integrar avaliação e intervenção no mesmo processo é antiga na literatura psicológica, podendo ser encontrada em diversos momentos e sob diferentes fundamentações teóricas (vide, por exemplo, Barbieri 2002; Finn \& Tonsager, 1992; Winnicott, 1971/1984). Apesar disso, no Brasil, apenas mais recentemente se observam esforços conjuntos para a descrição de fundamentos, propostas técnicas e seu teste empírico.

A partir dessas reflexões, os modelos apresentados a seguir se propõem a expandir o chamado modelo tradicional de Avaliação Psicológica, no qual predomina a compreensão de que avaliação e intervenção são processos distintos. Frente a isso, tais modelos se propõem a ajudar os pacientes/clientes diretamente, diferindo-se da AP tradicional. Para tanto, os avaliadores esperam tornar a experiência da avaliação significativa e provocar mudanças positivas nos pacientes e nas pessoas com participação em sua vida (como família ou terapeutas; Finn, 2017). Tais modelos, propostos para contextos clínicos, vêm sendo denominados como Psicodiagnóstico Interventivo (PI), Psicodiagnóstico Interventivo de Orientação Psicanalítica (PIOP) e Avaliação Terapêutica (na literatura internacional, Therapeutic Assessment - TA). Sua apresentação será feita na mesma ordem de seu aparecimento na literatura científica brasileira.

A partir de um organograma sobre as diferenças entre os modelos de AP em função de seus objetivos (Figura 1), Finn (2017) propõe que a avaliação pode ser considerada terapêutica quando é planejada para essa finalidade. Nesse contexto, Finn (2017) esclarece que o critério de colaboração trata dos esforços dos profissionais para envolver o cliente nas fases desse processo, quais sejam, 1. construção das razões para a avaliação, 2. observação das respostas dos testes ou comportamentos, 3. descoberta do significado desse conjunto de dados, 4. criação de recomendações úteis e 5 . formulação de resumos. $\mathrm{O}$ mesmo autor propõe como segundo critério o grau de estruturação do processo de avaliação, isto é, aos objetivos das entrevistas e padronização do uso de recursos de avaliação (instrumentos ou atividades, por exemplo). Como se mostrará a seguir, os modelos PI e PIOP se encaixam na categoria "não estruturada" da Figura 1 e o modelo TA, numa categoria separada.

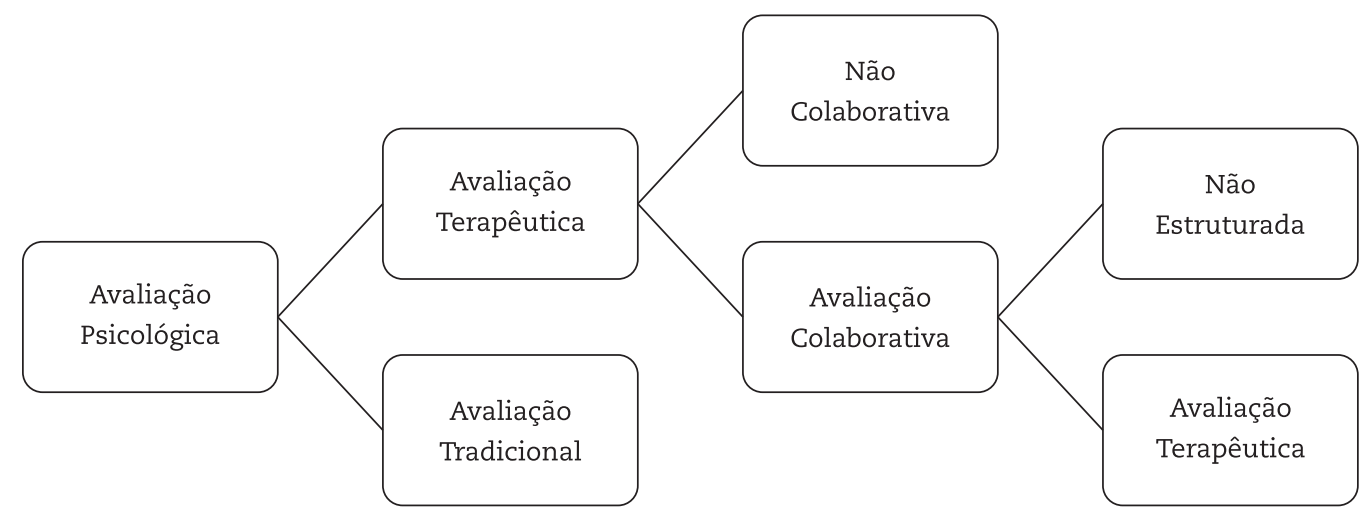

Figura 1. Organograma dos modelos de Avaliação Psicológica. Adaptado de Finn (2017)

Na primeira seção do artigo, os modelos são apresentados usando os seguintes critérios: 1. Fundamentos; 2. Estrutura, tipo de técnicas e intervenções usadas; 3. Vantagens da proposta em relação ao modelo tradicional de AP e 4. Pesquisas sobre o modelo. Em segui$\mathrm{da}$, os modelos são comparados em suas similaridades e diferenças; por fim, são propostas reflexões a partir dessa comparação, em termos da formação, pesquisa e aplicações de tais modelos no país.

Ao longo do artigo, propõe-se os termos "psicodiagnóstico" e "avaliação psicológica" como similares. Apesar de alguns autores os considerarem como distintos (Hutz 
et al., 2016), entende-se que, a rigor, ambos os conceitos se referem a um processo de coleta e análise de informações de diversas fontes, para fins de tomada de decisão (Conselho Federal de Psicologia [CFP], 2018). Ainda, tal escolha é feita com a intenção de promover a apropriação dos modelos aqui expostos por pesquisadores e profissionais aplicados de diferentes formações teóricas e contextos de atuação, visto que tais modelos podem ser estendidos para além do contexto clínico.

\section{O Psicodiagnóstico Interventivo (PI)}

Fundamentos. A proposta do Psicodiagnóstico Interventivo (PI) parece, à primeira vista, simples: conduzir processos de avaliação psicológica não apenas para realizar diagnósticos, mas também oferecer feedback, visando promover efeitos terapêuticos enquanto ocorre a avaliação. Uma análise mais detalhada, contudo, revela questionamentos epistemológicos, metodológicos e institucionais vindos das Psicologias Clínica e Social.

Ancona-Lopes (1995) situa a origem do PI a partir da análise do atendimento oferecido por serviços-escola de Psicologia nas décadas de 1980 e 1990 . Ao constatar o alto número de desistências e baixo número de altas, a mesma autora apontou para a falta de um projeto integrado de funcionamento desses serviços e dificuldades para definir alta e enfrentar limitações institucionais, marcadamente dos modelos mais tradicionais de atendimento e supervisão.

Esse movimento levou à busca por "modos inovadores de atuação psicológica" (Ancona-Lopes, 1995, p. 80), a fim de garantir treinamento de estagiários e oferecer atendimento qualificado. Ancona-Lopes (1995) afirma que essas mudanças acompanharam questionamentos sobre a função dos serviços-escola e dos aspectos sociais para compreensão dos casos atendidos. Desta forma, o PI se propôs como reflexão e ampliação da prática clínica da Psicologia, visando superar o mero "transporte de técnicas do consultório privado para a instituição" (Ancona-Lopes, 1995, p. 86). Ainda, ao questionar o status da Psicologia Clínica e dos serviços-escola predominantes na época, o PI foi proposto como meio de repensar a triagem e diagnóstico psicológicos, integrando a compreensão das relações institucionais e de classes sociais na busca por melhorias no atendimento.

A proposta do PI foi baseada, inicialmente, na Fenomenologia Existencial e na crítica a alguns pressupostos do psicodiagnóstico tradicional, em especial seu status de objetividade. Segundo Ancona-Lopez (1995), o psicodiagnóstico se apoiaria em conhecimentos incompletos e por vezes contraditórios, ao fazer uso de recursos baseados em diferentes teorias (como testes ou modelos de entrevista) ou para uma categorização diagnóstica pouco útil em termos terapêuticos, no sentido de ser pouco informativa ao paciente e não oferecer informações sobre as especificidades deste ao avaliador.
Estrutura, tipo de técnicas e intervenções usadas. O Psicodiagnóstico Grupal Interventivo serviu de base inicial ao PI (Ancona-Lopez, 1995). Nesse tipo de atendimento, grupos de pais e crianças com perfil semelhante de idade e queixas são atendidos por supervisor e estagiários em conjunto, visando compreender as dificuldades trazidas pelas famílias sob seus próprios pontos de vista, retirando o caráter passivo das pessoas atendidas nesse processo. Durante esses atendimentos, são realizadas atividades lúdicas com as crianças, além de subgrupos de famílias ou crianças e estagiários para entrevistas individualizadas, em geral num segundo momento, o que Ancona-Lopez (1995) chama de "técnica do cochicho". Tais atividades são planejadas para observar as crianças nesses contextos (grupal, subgrupal, com suas famílias), podendo incluir a aplicação de testes psicológicos padronizados, de acordo com dúvidas que surjam ao longo do processo. São feitos encontros intercalados com o grupo de crianças ou com seus responsáveis. Nos encontros com as crianças, o foco é de acolhimento e avaliação; já com os responsáveis o foco se volta para a reflexão sobre a criança, a demanda trazida e observações ao longo da avaliação. $\mathrm{O}$ atendimento dura entre três a oito meses, com frequência semanal.

Posteriormente, Ancona-Lopez (2013) descreveu atualizações do PI para atendimentos individualizados (uma criança e sua família), bem como de ações complementares (visitas domiciliares, escolares e observação do caderno escolar). No mesmo trabalho são descritos os fundamentos desse modelo tanto pela Psicologia Fenomenológica-Existencial quanto pela teoria psicanalítica. Nessa última teoria, Guimarães e Fantini (2013) apresentam uma fundamentação semelhante ao do Psicodiagnóstico Interventivo de Orientação Psicanalítica (PIOP), ainda que a estrutura e técnicas sejam mais parecidas com o PI do que com o PIOP.

No PI, os avaliadores assumem uma postura acolhedora e de incentivo para que os responsáveis pela criança possam refletir sobre os motivos da busca por ajuda e não apenas ofereçam informações. Para tanto, os avaliadores realizam grupos de famílias para discussão sobre os motivos da busca por atendimento, a fim de promover vínculos grupais, tirar dúvidas e desmistificar suas queixas como problemas individualizados. Com a "técnica do cochicho", propõem-se reflexões mais personalizadas sobre pontos da história de vida da criança, posturas e expectativas dos responsáveis quanto a elas e diferenças entre seus pontos de vista.

Nos grupos de crianças, as atividades realizadas servem de base para devolutivas parciais, ao final de cada sessão. Ancona-Lopez (1995) menciona que o que foi observado nessas atividades serve como base para discussão sobre a queixa trazida pela família (por exemplo, ao se comparar como esta abordou uma brincadeira e como aborda atividades escolares). Para se obter mais dados sobre a criança e sua família são realizadas visitas 
domiciliares e escolares (Ancona-Lopez, 2013). Tais observações são discutidas posteriormente com a criança e seus responsáveis em devolutivas parciais, nas quais são apresentadas e discutidas impressões sobre a demanda até aquele momento. No final do atendimento, são realizadas devolutivas usando técnicas lúdicas e um laudo psicológico entregue aos responsáveis, que é lido e discutido.

Vantagens da proposta em relação ao modelo tradicional de AP. O PI foi proposto para lidar com as limitações do modelo tradicional, particularmente, sua falta de coesão metodológica e teórica e sua baixa efetividade. Ainda, o mesmo modelo foi pensado como estratégia de atendimento em serviços-escola de Psicologia e treino de psicólogos-estagiários. Propõe-se, em relação ao modelo tradicional, um formato compreensivo de avaliação, focado na sensibilização e incentivo à autonomia dos responsáveis pela criança, promovendo a superação das dificuldades que levaram à busca por ajuda profissional.

Pesquisas sobre o modelo. Apesar de publicação recente, descrevendo atualizações do PI e apresentando teses e dissertações mais recentes sobre o tema (AnconaLopez, 2013), a maior parte da pesquisa com esse modelo se refere a teses e dissertações realizadas na década de 1980 citadas em Ancona-Lopez (1995) e apresentações em eventos científicos. Destes, destacam-se os Encontros de Serviços-Escola de Psicologia, que ocorrem no estado de São Paulo desde 1993. Em pesquisa nas bases SciELO, BVS e LILACS, o único trabalho posterior a AnconaLopez (2013) sobre o PI foi o de Evangelista (2016), que apresenta esse modelo, enfatizando seus fundamentos teóricos. Percebe-se que, apesar de o PI datar de esforços iniciados na década de 1980, estudos empíricos sobre seus potenciais e limitações são escassos e voltados para o atendimento de casos de crianças e suas famílias, tendo como principal contribuição, segundo Ancona-Lopez (1995; 2013), o fato de diminuir as filas de espera em serviços-escola, visto que esse atendimento seria suficiente para lidar com queixas de baixa gravidade.

\section{Psicodiagnóstico Interventivo de Orientação Psicanalítica (PIOP)}

Fundamentos. O PIOP pode ser definido como uma forma de avaliação terapêutica que trabalha com a dinâmica intrapsíquica do sujeito mediante o encontro com o avaliador (Paulo, 2005), buscando, simultaneamente, diagnosticar, compreender e intervir na problemática do indivíduo.

O PIOP é influenciado pelo Psicodiagnóstico Compreensivo (Trinca, 1984), com o qual o compartilha os objetivos de 1. elucidar o significado latente do sintoma; 2. enfatizar a dinâmica inconsciente do paciente e de sua família; 3 . buscar compreensão globalizada do paciente; 4. priorizar o julgamento clínico dos dados, implicando no uso dos recursos mentais do avaliador e 5. priorizar métodos e técnicas baseados na associação livre, utilizados como formas de entrevista, cujos resultados são avaliados por meio da livre inspeção do material (Barbieri, 2002).

A outra influência do PIOP são as Consultas Terapêtuticas (Winnicott, 1971/1984), nas quais se combina avaliação e intervenção. Segundo esse autor, nas primeiras sessões, há emersão de aspectos verbais e não verbais relacionados ao motivo da consulta que levariam muito tempo para vir à tona novamente em uma psicoterapia posterior. Por esse motivo, torna-se crucial o uso de intervenções desde o contato inicial com o paciente. Dentro desse contexto, na clínica infantil, Aberastury (1979/1982) apontou para a importância do primeiro contato com a criança na hora de jogo, na qual surgem fantasias inconscientes de enfermidade e de cura.

Sendo assim, o PIOP almeja, desde os primeiros encontros com o paciente, integrar aspectos dissociados da sua personalidade (Barbieri, 2008, 2009, 2010; Winnicott, 1971/1984) por meio do vínculo com o avaliador. Para tanto, este último adota uma postura de confiabilidade e previsibilidade, favorecendo a ocorrência do que Winnicott chamou de ilusão. O desenvolvimento do processo ilusório, por sua vez, permite estabelecer um espaço potencial na qual acontecem os fenômenos transicionais e o gesto espontâneo, ou seja, o caminho para a autenticidade. Dessa forma, o propósito principal do PIOP não consiste em esbater sintomas, mas sim auxiliar o paciente na compreensão do sentido deles, a fim de reconhecer e remover os bloqueios que interferem no processo de amadurecimento emocional.

Estrutura, tipo de técnicas e intervenções usadas. O PIOP adota as contribuições teórico técnicas de Winnicott para interpretação do material produzido pelo paciente nos encontros. Todavia, não há um procedimento uniforme a ser seguido, já que ele está ligado ao pensamento clínico do avaliador. Dessa forma, não se pode estipular passos a serem seguidos, tampouco o número de encontros necessários. Contudo, outros estudos descrevem posturas diversas, desde a utilização de duas sessões (entrevista inicial e devolutiva; Trinca, 2003) até doze encontros, incluindo o uso da testagem (Heck, 2014, 2018; Paulo, 2005), se o avaliador ponderar que seja importante para o processo.

Apesar de não haver passos pré-determinados no PIOP, pode-se referir algumas técnicas e intervenções utilizadas ao longo do processo. Conforme mencionado, o material verbal e não verbal expresso pelo paciente desde os primeiros encontros é base para intervenções de diversas naturezas (holding, perguntas, assinalamentos, interpretações, por exemplo). Vale alertar que sempre se deve levar em conta o que o paciente pode suportar ouvir naquele momento. Mesmo assim, se houver "erros" de interpretação, a busca por compreender o paciente faz com que ele sinta que está sendo acolhido e ouvido por alguém que busca ajudá-lo (Heck \& Barbieri, 2017).

Como já referido, o avaliador prioriza no PIOP o uso de métodos e técnicas de exame com base na 
associação livre, tal como os testes projetivos e os procedimentos clínicos de investigação pouco ou não estruturados. Ressalta-se que, nesse caso, a avaliação desses procedimentos é realizada por meio da livre inspeção do material produzido, baseada na experiência do psicólogo (Trinca, 1984). A despeito disso, não há o impedimento do uso de instrumentos padronizados como as escalas psicométricas. Nesse sentido, o clínico deve estar atento para a administração padronizada desses instrumentos, conforme seus manuais, integrando seus resultados à luz dos demais achados (Heck \& Barbieri, 2017). Por outro lado, baseando-se na utilização do jogo do rabisco por Winnicott (1971/1984) como uma técnica projetiva para auxiliar a comunicação com o paciente, o PIOP usa instrumentos como mediadores da comunicação entre paciente e avaliador. Dessa forma, a flexibilidade nos processos de administração, levantamento e interpretação do material permitem compreensão da singularidade do paciente (Heck \& Barbieri, 2017).

$\mathrm{Na}$ linguagem Winnicottiana, pode-se dizer que as técnicas e métodos projetivos fomentam a experiência de ilusão e de transicionalidade. Desse modo, dentro dos limites do setting, o paciente vivencia um funcionamento mental mais arcaico em que as fronteiras entre o self e a realidade exterior se tornam mais fluidas (Heck \& Barbieri, 2017). Essa experiência é crucial para o estabelecimento de uma comunicação verdadeira com o mundo; portanto, o uso de instrumentos de avaliação e discussão de seus resultados pode ser terapêutico por si só, como observado também em contextos tradicionais de AP (Hutz el al., 2016).

No atendimento a crianças e adolescentes, o PIOP visa à avaliação da qualidade do ambiente no qual a criança vive à semelhança das consultas terapêuticas (Heck \& Barbieri, 2017). Para Winnicott (1971/1984), a inclusão da família nos atendimentos possibilita o acesso a suas fantasias, defesas e angústias, além de auxiliá-los a oferecer um ambiente suficientemente bom para os filhos.

Vantagens da proposta em relação ao modelo tradicional. O PIOP consiste num método que engloba avaliação e intervenção ao longo do processo, o que o torna terapêutico em si mesmo. Nesse contexto são trabalhadas devolutivas com o paciente durante todo o processo e não somente no final dele (como ocorre no psicodiagnóstico tradicional), promovendo efeitos terapêuticos desde o contato inicial com o paciente. A entrevista final no PIOP tem o propósito de síntese dos encontros ocorridos; nesse momento, o material trabalhado previamente é retomado, permitindo refletir sobre a experiência vivida em cada encontro.

Vale ressaltar que existem efeitos terapêuticos que também podem surgir no psicodiagnóstico tradicional, mas esses são compreendidos como sendo involuntários. Frente a isso, no PIOP busca-se realizar intervenções com o propósito da retomada, pelo paciente, de aspectos do seu desenvolvimento mediante as vivências transicionais. Outro ponto a ser considerado no PIOP em relação ao modelo tradicional diz respeito à compreensão do sentido dos sintomas pelo paciente, buscando-se não somente remiti-los, mas remover bloqueios ao processo de amadurecimento emocional do indivíduo rumo ao gesto espontâneo.

Pesquisas sobre o modelo. O PIOP é um método relativamente novo em nosso país e, portanto, carece de mais pesquisas (Milani, Tomael, \& Greinert, 2014). Todavia, podem-se citar alguns trabalhos importantes sobre o tema. Barbieri $(2002 ; 2017)$ trabalhou com crianças com tendência antissocial e Heck $(2014 ; 2018)$ atendeu seis crianças com sintomas de ansiedade e seus pais; Trinca (2003) atendeu quinze crianças em situação de pré-cirurgia; Loli, Abrão e Tardivo (2016) apresentam um estudo com adolescentes em psicoterapia. Com adultos, há estudos do PIOP com pacientes deprimidos (Paulo, 2005), com obesidade grau II (Mishima, 2011) e idosos (Gil, 2010; Salles \& Tardivo, 2017). Tardivo (2007) descreveu o ensino do PIOP para alunos de graduação e seus desafios. Os resultados dessas pesquisas demonstraram que os participantes apresentaram ganhos terapêuticos decorrentes do método adotado.

\section{Avaliação Terapêutica}

Fundamentos. A Therapeutic Assessment (TA) começou a ser sistematizada por Stephen Finn na década de 1980. Segundo Finn e Martin (2013), o primeiro estudo controlado evidenciando benefícios para avaliação dos clientes foi o de Finn e Tonsager (1992). Desde então, a TA tem sido amplamente difundida nos EUA, continente Europeu e Japão (vide o site do Therapeutic Assessment Institute, https://www.therapeuticassessment.com/). Mais recentemente, a TA passou a ser difundida no Brasil, havendo até o momento pouca publicação realizada por pesquisadores brasileiros (Taub \& Cecchini, 2014; Vieira, 2017; Villemor-Amaral, 2016; Villemor-Amaral \& Scortegagna, 2018) e a tradução do livro de Finn (2017), que descreve o modelo com mais detalhes.

O modelo desenvolvido por Finn foi motivado, dentre outras razões, pela sua curiosidade quanto às possibilidades de uma avaliação psicológica gerar mudanças positivas na vida das pessoas. Em suas buscas, o autor se deparou com contribuições da Psicologia Humanista, conforme proposta de Fischer (1985/1994) que referia que o processo de avaliação fosse mais humano, respeitoso e compreensível para os clientes, propondo fundamentos e técnicas de avaliação colaborativa. Esses pressupostos alinhavam-se com os princípios defendidos por Finn e Tonsager (1992) de respeito para com o cliente, redução da assimetria entre terapeuta e clientes, e diálogo com eles sobre os resultados dos testes. Ainda que a TA possa ser praticada por profissionais de diferentes orientações teóricas, sua fundamentação está pautada nas teorias intersubjetivas, fenomenológicas e interpessoais (Finn \& Chudzik, 2010). 
Dessa forma, Finn (2017) descreve características essenciais desse processo, a saber: 1. colaboração, já que o cliente é convidado a participar ativamente de todo o processo; 2. curiosidade, que envolve um movimento constante do cliente e o avaliador para saber mais sobre as questões trabalhadas durante o processo; 3 . compaixão, no sentido de solidariedade com a dor do cliente; 4. humildade, ao compreender que, nesse processo, ambos o cliente e o terapeuta têm saberes de igual importância; 5. abertura, na medida em que é preciso que tanto terapeuta quanto cliente estejam disponíveis para o processo; e 6. respeito do terapeuta para acolher a fala do cliente, livre de julgamentos. Finn e Martin (2013) sugerem que a Psicologia reconheça que o ser humano é intencional e resoluto, e que o conhecimento/entendimento dos profissionais não é mais real ou válido do que o dos clientes.

Estrutura, tipo de técnicas e intervenções usadas. A TA é concebida como um processo semiestruturado de seis passos, a saber, 1. sessões iniciais; 2. sessões com testes padronizados; 3 . sessões de avaliação interventiva; 4 . sessões de sumário/discussão; 5 . fornecimento de feedback escrito e 6. follow-up (Finn, 2017). Cada uma dessas etapas é descrita a seguir.

$\mathrm{Na}$ etapa das sessões iniciais, a meta é ajudar o cliente a se sentir acolhido, seguro e aceito. Para isso, o cliente é convidado a colaborar e formular questões que vão guiar a avaliação. A elaboração dessas questões de forma conjunta contribui para diminuir a ansiedade (ao definir o foco da avaliação) e engajar o cliente na reflexão sobre si, despertando sua curiosidade e fazendo-o pensar e falar sobre suas histórias. Por sua vez, essa reflexão fornece "portas" ao avaliador, para que o cliente possa ser alcançado, sendo importante escutar cuidadosamente possíveis questões importantes para o cliente (Finn, 2017).

Criar as perguntas pode parecer fácil em um primeiro momento. Contudo, há diversos processos psicológicos envolvidos, de forma que conseguir fazer tais perguntas é um passo importante para compreender o cliente. Ao concentrar-se em questões específicas, os clientes definem preocupações e prioridades, o que os ajuda a se motivar a passar pela etapa de responder aos testes de forma aberta e honesta. Além disso, tais questões permitem identificar caminhos considerados difíceis pelo cliente e a avaliar e desenvolver curiosidade e auto-observação, o que por si só pode reduzir o sofrimento e preparar o caminho para a mudança terapêutica (Finn \& Chudzik, 2010). Assim, é importante que as perguntas criadas sejam factíveis de serem respondidas, envolvam e façam sentido ao próprio cliente. A todo momento é preciso que o terapeuta transmita confiança que poderá ajudar, demonstrando boa vontade em admitir que não sabe de tudo, mas que vai trabalhar para tanto.

A etapa inicial, necessariamente, é seguida pelas sessões em que testes padronizados são administrados. Essa etapa dura três ou quatro sessões, com um teste em geral por encontro (Finn \& Chudzik, 2010). A escolha dos testes é determinada principalmente pela natureza das perguntas dos clientes (ou solicitantes da avaliação), também dependendo do treinamento, experiência e preferências pessoais do avaliador, isto é, não há bateria de testes predeterminada.

Embora não haja muitas diferenças durante a aplicação dos testes padronizados na TA e na avaliação tradicional, Finn (2017) reforça a importância de seguir a padronização do teste e propõe iniciar com a administração dos testes que estão mais próximos da pergunta central do cliente. Assim, ele introduz cada teste de acordo com sua relevância para a questão do cliente, dedicando esforço para explicar a importância deles, de modo a reduzir a ansiedade do cliente ao mostrar que está colhendo informações relevantes para o atendimento.

Ao término dessa etapa, o avaliador pergunta sobre a experiência do cliente ao realizar a tarefa, prestando atenção especial a eventos que pareçam relacionados às questões que o cliente formulou. O diálogo durante a avaliação é útil para ajudar o avaliador a entender o comportamento do cliente e os escores dos testes. Segundo Finn (2017), os clientes valorizam as informações dos testes para compreender as "peças de seus quebra-cabeças".

A terceira etapa corresponde às sessões de intervenção, sendo uma versão padronizada do que Fischer (1985/1994) chamou de "avaliação do processo", na qual o avaliador provoca o diálogo sobre os problemas dos clientes, muitas vezes utilizando materiais de teste. A sessão de intervenção é uma importante ferramenta para impactar a história do cliente sobre ele mesmo e o mundo, além de ajudar a prever possíveis soluções às dificuldades inerentes aos seus problemas.

A quarta etapa corresponde às sessões de discussão. Finn (2017) organiza os resultados em três níveis. No nível 1, são considerados os resultados que verificam os modos habituais de pensar sobre si dos clientes, que serão facilmente identificados e aceitos por eles. Os resultados de Nível 2 são aqueles que modificam ou amplificam os modos de pensar dos clientes, mas que podem ameaçar a sua autoestima ou autopercepção. Por fim, os resultados do nível 3 são bastante diferentes dos modos de pensar dos clientes, podendo ser rejeitados por eles. Normalmente, os resultados do Nível 3 estão relacionados à ansiedade dos clientes, podendo mobilizar seus mecanismos de defesa e, por isso, é mais indicado que sejam trabalhados em terapias de longo prazo. Segundo Finn (2017), ao iniciar uma sessão de discussão de avaliação com informações de nível 1e movendo-se gradualmente às conclusões de Nível 2, cria-se a condição ideal para os clientes incorporarem novas informações sobre as formas como eles pensam sobre si mesmos e o mundo.

$\mathrm{Na}$ quinta etapa são fornecidos feedbacks escritos. Finn (2017) descreve a influência de autores humanistas sobre o tema, sugerindo que o relatório seja produzido 
no formato de carta, na qual se referem às hipóteses e os resultados da experiência, para que o cliente compreenda o processo e que outros profissionais envolvidos no tratamento possam ser ajudados. Além disso, os clientes podem contribuir com a reflexão sobre os pontos descritos na carta. Nessa carta, o avaliador deve evitar formalismos e o uso de terminologia psiquiátrica e precisa considerar a experiência de interação com o cliente.

A etapa 6 corresponde às sessões de follow-up (acompanhamento). Assim, é útil se reunir novamente com o cliente após dois ou três meses para conversar sobre o processo avaliativo e discutir quaisquer questões ou desenvolvimentos que surgiram nesse período. Tal oportunidade parece especialmente valiosa para clientes que não façam psicoterapia no final da avaliação.

Vantagens da proposta em relação ao modelo tradicional. A TA integra pesquisa e teoria nas áreas de apego, desenvolvimento infantil, neurobiologia, psicopatologia e psicoterapia, revolucionando a maneira como se pensa e interage com os clientes que procuram serviços psicológicos e sugerindo que 1 . a relação entre cliente e avaliador é mais importante do que foi reconhecido nas perspectivas tradicionais de avaliação; 2 . testes de personalidade como o Rorschach e o Teste de Apercepção Temática são muito úteis por estimular o hemisfério direito e o funcionamento cerebral subcortical, fornecendo informações que os clientes não podem relatar diretamente e 3. quando as avaliações psicológicas fornecem aos clientes experiências emocionais poderosas, promovem-se mudanças terapêuticas (Finn, 2012).

Acredita-se, desse modo, que a TA incorporou uma cadeia de conhecimentos para produzir uma abordagem valida e com suporte empírico para promover mudanças pessoais. Ela se embasa na facilitação de insights significativos que são eficientemente disponíveis usando recursos de AP, a fim de promover um processo de autodescoberta colaborativo, respeitoso, gentil, experiencial e de apoio (Finn \& Martin, 2013).

Pesquisas sobre o modelo. Há um conjunto de estudos empíricos que sugerem os benefícios de uso da TA. Pacientes adultos têm demonstrado redução nos sintomas e aumento da autoestima e da esperança (Finn \& Tonsager, 1992). Em comparação com as avaliações tradicionais, a avaliação colaborativa também levou a melhor adesão ao tratamento recomendado (Ackerman, Hilsenroth, Baity, \& Blagys, 2000), e melhor aliança em psicoterapias subsequentes (Hilsenroth, Peters, \& Ackerman, 2004). Além das pesquisas empíricas, uma série de estudos de caso foram relatados por Finn (2017) e outros autores, com adultos (Aschieri \& Smith, 2012; Fischer \& Finn, 2008; Peters, Handler, White, \& Winkel, 2008; Wygant \& Fleming, 2008), crianças (Aschieri, Fantini, \& Bertrando, 2012; Fantini, Aschieri, \& Bertrando, 2013; Tharinger et al, 2012), adolescentes (Austin, Krumholz, \& Tharinger, 2012; Michel, 2002) e casais (Smith, Nicholas, Handler, \& Nash, 2011).

\section{Desafios para Uso dos Modelos e Perspectivas Futuras no Brasil}

Os três modelos aqui estudados compartilham do pressuposto de que é possível promover efeitos terapêuticos usando recursos de AP, superando limitações do modelo tradicional dessa área. Os processos devem ser compreensivos e promotores de insight. Para tanto, as entrevistas com os envolvidos servem para promover o desenvolvimento e/ou aprimoramento de recursos de enfrentamento e superação das dificuldades relatadas.

Ancona-Lopez (2013) descreve mudanças psicossociais nas últimas décadas entendidas como desafios, não apenas para o PI, mas para avaliação de crianças e famílias em geral. O mesmo trabalho, contudo, não discutiu desafios técnicos ou metodológicos relativos ao PI, sugerindo que, perante as mudanças descritas, o principal desafio se refere à postura profissional e ética. Em nossa experiência prévia como supervisores usando esse modelo, observamos que o principal desafio relativo ao PI se refere à qualidade do treino básico para uso do modelo, especialmente sua operacionalização, ou seja, o uso de recursos tradicionais de AP em conjunto com entrevistas, observações e devolutivas parciais. Devido a seu caráter predominantemente idiográfico, não existe uma padronização de instrumentos e atividades, demandando dos avaliadores o domínio de conceitos de Psicologia Fenomenológico-Existencial, Psicanálise, entrevista e observação clínicas e instrumentos padronizados de AP. Além disso, não há estudos empíricos continuados sobre o PI com grandes amostras ou grupos clínicos em especial. Por outro lado, o PI se encontra consolidado na Universidade Paulista (UNIP), sendo relevante especialmente no estado de São Paulo, onde se concentra a maior parte dos cursos de Psicologia dessa instituição.

No caso do PIOP, a difusão do modelo ainda é um desafio para os pesquisadores na área. Contudo, existem algumas pesquisas recentes e conduzidas por diferentes autores que têm demonstrado resultados positivos no Brasil (Barbieri, 2017; Mishima, 2011; Heck, 2014). É importante ressaltar que, devido à complexidade desse modelo, a postura do profissional e a qualidade do seu mundo mental são ferramentas fundamentais para, permitindo fornecer holding ao paciente e fomentar um ambiente facilitador de contato genuíno e expressivo do verdadeiro self. Diante dessa demanda, a busca de supervisão e psicoterapia pessoal são condições intrínsecas para realizar esse modelo.

Com relação à TA, pesquisas recentes, ainda que iniciais no país, apontam para resultados promissores em termos de seus efeitos terapêuticos e possibilidades de treinamento. O treinamento, assim como para o PI e ao PIOP, é complexo e demanda diversas habilidades. Villemor-Amaral (2016) reforça que, mesmo a TA tendo um processo semiestruturado, requer sensibilidade e criatividade do psicólogo para se adequar à singularidade e à relação estabelecida com cada cliente. 
Nas propostas dos três modelos, observa-se o indicativo de que processo deva ser breve e depende de uma atitude colaborativa das pessoas avaliadas. Ancona-Lopez (2013), por exemplo, assevera que o PI seria adequado para queixas pontuais e de baixa gravidade, comuns nas filas de espera de serviços-escola de Psicologia, visto ser uma intervenção breve e focada no incentivo à autonomia (denotando que isso seria mais difícil para pessoas que demandam acompanhamento prolongado). Barbieri (2002; 2008) observou que o PIOP promove mudanças mais consistentes no funcionamento de pessoas com estruturas neuróticas do que em pessoas com estruturas psicóticas de personalidade. Finn (2017) propõe uma distinção entre níveis de resultados, sugerindo que aqueles mais relacionados às ansiedades do cliente possam ser difíceis de serem assimilados e demandem longo período de elaboração.

É importante reforçar que por depender de uma atitude colaborativa ao longo de todo o processo, os três modelos aqui discutidos são contraindicados para situações de avaliação compulsória, para fins de seleção e/ou somente classificação diagnóstica ou em que a avaliação possa ser usada contra as pessoas avaliadas. A comparação aqui apresentada permite afirmar que os três modelos apresentam potencial para usos inovadores da AP, assim como da promoção de estratégias de intervenção psicológica com fundamentação teórica e empírica coesa. Entende-se que tal possibilidade se estende não apenas à área de Avaliação Psicológica, mas à Psicologia brasileira como um todo.

\section{Referências}

Aberastury, A. (1982). Psicanálise da criança: Teoria e técnica (A. L. L. de Campos, trad.). Porto Alegre, RS: Artmed. (Trabalho original publicado em 1979).

Ackerman, S. J., Hilsenroth, M. J., Baity, M. R., \& Blagys, M. D. (2000). Interaction of Therapeutic Process and Alliance During Psychological Assessment. Journal of Personality Assessment, 75(1), 82-109. doi 10.1207/S15327752JPA7501_7

Ancona-Lopez, M. (1995). Psicodiagnóstico: processo de intervenção. São Paulo: Cortez.

Ancona-Lopez, S. (2013) Psicodiagnóstico interventivo: Evolução de uma prática. São Paulo: Cortez.

Aschieri, F., \& Smith, J. D. (2012). The effectiveness of therapeutic assessment with an adult client: A single-case study using a time-series design. Journal of Personality Assessment, 94(1), 1-11. doi: 10.1080/00223891.2011.627964

Aschieri, F., Fantini, F., \& Bertrando, P. (2012). Therapeutic Assessment with children in family therapy. Australian and New Zealand Journal of Family Therapy, 33(04), 285-298. doi: 10.1017/aft.2012.37

Austin, C., Krumholz, L., \& Tharinger, D. J. (2012). Therapeutic Assessment with an adolescent: Choosing connections over substances. Journal of Personality Assessment, 94(6), 571-585. doi: 10.1080/00223891.2012.670679

Barbieri, V. (2002). A família e o psicodiagnóstico como recursos terapêuticos no tratamento dos transtornos de conduta infantis (Tese de doutorado). Departamento de Psicologia Clínica, Instituto de Psicologia da Universidade de São Paulo, São Paulo.

Barbieri, V. (2008). Por uma ciência-profissão: O psicodiagnóstico interventivo como método de investigação científica. Psicologia em Estudo, 13(3), 575- 584. doi: 10.1590/S1413-73722008000300019

Barbieri, V. (2009). O psicodiagnóstico interventivo psicanalítico na pesquisa acadêmica: Fundamentos teóricos, científicos e éticos. Boletim de Psicologia, 59(131), 209-222. Recuperado de http://pepsic.bvsalud.org/pdf/bolpsi/v59n131/v59n131a07.pdf

Barbieri, V. (2010). Psicodiagnóstico tradicional e interventivo: Confronto de paradigmas? Psicologia: Teoria e Pesquisa, 26(3), 505-513. doi: 10.1590/S0102-37722010000300013.

Barbieri, V. (2017). O Psicodiagnóstico Interventivo Psicanalítico na tendência antissocial. A família e as técnicas projetivas como recursos terapêuticos no atendimento de crianças. Balti, Republic of Moldova:Novas Edições Acadêmicas.

Borsa, J. C. (2016). Considerações sobre a formação e a prática em avaliação psicológica no Brasil. Temas Em Psicologia, 24(1), 131-143. doi: 10.9788/TP2016.1-09

Conselho Federal de Psicologia, CFP. (2018). Resolução No 9, de 25 de abril de 2018. Brasília: Conselho Federal de Psicologia.

Damásio, B. F., \& Borsa, J. C. (Eds.) (2018). Manual de Desenvolvimento de Instrumentos Psicológicos. São Paulo: Vetor.

Evangelista, P. (2016). O psicodiagnóstico interventivo fenomenológico-existencial grupal como possibilidade de ação clínica do psicólogo. Revista da Abordagem Gestáltica, 22(2), 219-224. Recuperado de http://pepsic.bvsalud.org/pdf/rag/v22n2/v22n2a14.pdf

Fantini, F., Aschieri, F., \& Bertrando, P. (2013). "Is our daughter crazy or bad?”: A case study of Therapeutic Assessment with children. Contemporary Family Therapy, 35(4), 731-744. doi: 10.1007/s10591-013-9265-3

Finn, S. E. (2012). Implications of recent research in neurobiology for psychological assessment. Journal of Personality Assessment, 94(5), 440449. doi: 10.1080/00223891.2012.700665

Finn, S. E. (2017). Pela perspectiva do cliente. Teoria e técnica da Avaliação Terapêutica. São Paulo: Hogrefe.

Finn, S. E., \& Chudzik, L. (2010). L'Evaluation Thérapeutique: Une intervention originale brève. EmS. Sultan \& L. Chudzik (Eds.), Du diagnostic au traitement: Rorschach et MMPI-2 (pp. 203-226). Wavre, Belgium: Mardaga.

Finn, S. E., \& Martin, H. (2013). Therapeutic Assessment: Using psychological testing as brief therapy. Em K. F. Geisinger, B. A. Bracken, J. F. Carlson, J. I. C. Hansen, N. R. Kuncel, S. P. Reise, \& M. C. Rodriguez (Eds.). APA handbook of testing and assessment in psychology, Vol. 2: Testing and assessment in clinical and counseling psychology (pp. 453-465). Washington, DC: American Psychological Association.

Finn, S. E., \& Tonsager, M. E. (1992). Therapeutic effects of providing MMPI-2 test feedback to college students awaiting therapy. Psychological Assessment, 4(3), 278-287. doi: 10.1037/1040-3590.4.3.278

Fischer, C. T. (1994). Individualizing psychological assessment. Hills-dale, NJ: Erlbaum (Trabalho original publicado em 1985). 
Fischer, C. T., \& Finn, S. E. (2008). Developing the life meaning of psychological test data: Collaborative and therapeutic approaches. Em R. P. Archer \& S. R. Smith (Eds.), Personality assessment (pp. 379-404). New York, NY: Routledge.

Gil, C. A. (2010). Recordação e transicionalidade: A oficina de cartas, fotografias e lembranças como intervenção terapêutica grupal com idosos (Tese de doutorado). Instituto de Psicologia, Universidade de São Paulo, São Paulo. Recuperado de http://www.teses.usp.br/teses/ disponiveis/47/47133/tde-20012011-111211/pt-br.php

Guimarães, G., \& Fantini, M. N. A. (2013). Movimentos transferenciais no psicodiagnóstico interventivo. Em Ancona-Lopez, S. (2013). Psicodiagnóstico interventivo: Evolução de uma prática (pp. 77-89). São Paulo: Cortez.

Heck, V. (2014). A influência das invasões ambientais nos sintomas de ansiedade infantil: Uma experiência de psicodiagnóstico interventivo (Tese de doutorado). Instituto de Psicologia, Universidade Federal do Rio Grande do Sul.

Heck, V.S., \& Barbieri, V. (2017). O Psicodiagnóstico Interventivo: Novos rumos na avaliação psicológica. Em Lins, M.R.C., Borsa, J. (Ed.), Avaliação psicológica: Aspectos teóricos e práticos. $1^{\text {a }}$ Ed., v.1, p. 458-469. Petrópolis: Vozes.

Heck, V. (2018). O Psicodiagnóstico Interventivo Psicanalítico na infância. Uma modalidade de avaliação psicológica terapêutica em crianças com sintomas de ansiedade. Balti - Moldávia: Novas Edições Acadêmicas.

Hilsenroth, M. J., Peters, E. J., \& Ackerman, S. J. (2004). The Development of Therapeutic Alliance During Psychological Assessment: Patient and Therapist Perspectives Across Treatment. Journal of Personality Assessment, 83(3), 332-344. doi: 10.1207/s15327752jpa8303_14

Hutz, C. S., Bandeira, D. R., \& Trentini, C. M. (Ed.) (2018). Avaliação da Inteligência e da personalidade. Porto Alegre: Artmed.

Hutz, C. S., Bandeira, D. R., Trentini, C. M., \& Krug, J. (Org.) (2016). Psicodiagnóstico. Porto Alegre: Artmed.

Loli, M. S. A., Abrão, J. L. F., \& Tardivo, L. S. L. P. C. (2016). O uso da imaginação no psicodiagnóstico e na psicoterapia de adolescentes. Boletim de Psicologia, 66(144), 80-97. Recuperado de http://pepsic.bvsalud.org/pdf/bolpsi/v66n144/v66n144a08.pdf

Michel, D. M. (2002). Psychological assessment as a therapeutic intervention in patients hospitalized with eating disorders. Professional Psychology: Research and Practice, 33(5), 470-477. doi: 10.1037/0735-7028.33.5.470

Milani, G. R., Tomael, M. M., \& Greinert, B. R. M (2014). Psicodiagnóstico interventivo psicanalítico. Estudos Interdisciplinares em Psicologia, 5(1), 80-95. Recuperado de http://pepsic.bvsalud.org/pdf/eip/v5n1/a06.pdf

Mishima, F. K. T. (2011). Obesidade feminina: Considerações a partir do psicodiagnóstico interventivo (Tese de Doutorado). Faculdade de Filosofia, Ciências e Letras de Ribeirão Preto, Universidade de São Paulo.

Paulo, M. L. L. de. (2005). Depressão e psicodiagnóstico interventivo: Proposta de atendimento. São Paulo: Vetor.

Peters, E. J., Handler, L., White, K., \& Winkel, J. (2008). “Am I going crazy, doc?”: A self-psychology approach to Therapeutic Assessment. Journal of Personality Assessment, 90(5), 421-434. doi: 10.1080/00223890802248679

Salles, R. J., \& Tardivo, L. S. L. P. C. (2017). Contribuições do pensamento de Winnicott para teoria e prática do psicodiagnóstico psicanalítico. Boletim - Academia Paulista de Psicologia, 37(93), 282-310. Recuperado de http://pepsic.bvsalud.org/pdf/bapp/v37n93/ v37n93a07.pdf

Santos, A. M. dos, Anache, A. A., \& Santana, R. C. de. (2015). Overview of Brazilian Scientific Production in Psychological Evaluation. Psico-USF, 20(3), 547-559. doi: 10.1590/1413-82712015200315

Smith, J. D., Nicholas, C. R. N., Handler, L., \& Nash, M. R. (2011). Examining the potential impact of a family session in therapeutic assessment: A single-case experiment. Journal of Personality Assessment, 93,204-212. doi: 10.1080/00223891.2011.559497

Tardivo, L. S. P. C. (2007). Psicodiagnóstico interventivo: Uma proposta de ensino em Atendimento Clínico. Mudanças - Psicologia da Saúde, 15(2), 128-134. doi: 10.15603/2176-1019/mud.v15n2p128-134

Taub, A., \& Cecchini, M. A. (2014). Avaliação Neuropsicológica: Modelos teóricos. Revista Neuropsicologia Latinoamericana, 6(3), 1-3. doi: 10.5579/rnl.2013.0205

Tharinger, D. J., Finn, S. E., Arora, P., Judd-Glossy, J., Ihorn, S. M., \& Wan, J. T. (2012). Therapeutic assessment with children: intervening with parents "behind the mirror". Journal of Personality Assessment, 94(2), 111-123. doi: 10.1080/00223891.2011.645932.

Trinca, A. M. T. (2003). A intervenção terapêutica breve e a pré-cirurgia infantil. São Paulo: Vetor.

Trinca, W. (1984). Processo diagnóstico de tipo compreensivo. Em W. Trinca (Eds.), Diagnóstico psicológico: A prática clínica (pp. 14-24). São Paulo, SP: EPU.

Vieira, P. G. (2017). Avaliação Terapêutica: Uma nova abordagem para o Psicodiagnóstico. Em P. Landin, R. Almeida \& A. Roma, (Eds.). Avaliação Psicológica: A atualidade da prática profissional (p. 21-31). São Paulo: Leader.

Villemor-Amaral, A. E. (2016). Perspectivas para a avaliação terapêutica no Brasil. Avaliação Psicológica, 15(2),249-255. Recuperado de http:// pepsic.bvsalud.org/pdf/avp/v15n2/v15n2a14.pdf

Villemor-Amaral, A. E., \& Scortegagna, S. A. (2018) Avaliação terapêutica na clínica com crianças, adolescentes e famílias. Em M. R. C. Lins, M. Muniz \& L. M. Cardoso (Eds.) Avaliação Psicológica infantil (115-128). São Paulo: Hogrefe.

Winnicott, D. W. (1984). Consultas terapêuticas em psiquiatria infantil (J. M. X. Cunha, Trad.). Rio de Janeiro: Imago. (Trabalho original publicado em 1971).

Wygant, D. B., \& Fleming, K. P. (2008). Clinical utility of the MMPI-2 Restructured Clinical (RC) Scales in therapeutic assessment: a case study. Journal of Personality Assessment, 90(2), 110-188. doi: 10.1080/00223890701845112

recebido em agosto de 2018 aprovado em janeiro de 2019

\section{Sobre os autores}

Alessandro Antonio Scaduto é psicólogo e doutor em Psicologia pela Faculdade de Filosofia Ciências e Letras de Ribeirão Preto Universidade de São Paulo (FFCLRP-USP). Atualmente, é Professor Adjunto do Departamento de Psicologia - Universidade Federal do Paraná (DEPSI-UFPR), na área de Avaliação Psicológica no contexto da Saúde.

Lucila Moraes Cardoso é psicóloga e doutora em Psicologia (área de concentração Avaliação Psicológica) pela Universidade São Francisco (USF). Atualmente é Professora Adjunta do curso de Psicologia e do Programa de Pós-graduação em Educação da Universidade Estadual do Ceará (UECE) e Colaboradora no Programa de Pós-graduação em Psicologia da Universidade Federal do Ceará (UFC).

Vanessa Stumpf Heck é psicóloga e doutora em Psicologia pela pela Universidade Federal do Rio Grande do Sul. Atualmente, realiza pós-doutorado na Faculdade de Filosofia Ciências e Letras de Ribeirão Preto - Universidade de São Paulo (FFCLRP-USP) e é coordenadora de cursos no Grupo-DOM (Porto Alegre - RS). 